\title{
What Determines the Drop Size in Sprays?
}

\author{
Stefan Kooij, ${ }^{1}$ Rick Sijs, ${ }^{1}$ Morton M. Denn, ${ }^{2}$ Emmanuel Villermaux, ${ }^{3,4}$ and Daniel Bonn ${ }^{1}$ \\ ${ }^{1}$ Van der Waals-Zeeman Institute, University of Amsterdam, Science Park 904, Amsterdam, Netherlands \\ ${ }^{2}$ Benjamin Levich Institute, City College of New York, CUNY, New York, New York 10031, USA \\ ${ }^{3}$ Aix Marseille Université, CNRS, Centrale Marseille, IRPHE UMR 7342, 13384 Marseille, France \\ ${ }^{4}$ Institut Universitaire de France, 75005 Paris, France
}

(Received 29 September 2017; revised manuscript received 8 February 2018; published 20 July 2018)

In many instances, sprays are formed from the breakup of liquid jets or sheets. We investigate the different parameters that determine the characteristic drop size in the breakup of sheets. We vary both the spraying parameters, such as the pressure and geometry of the nozzle, and the fluid parameters, such as viscosity and surface tension. The combined results show that the drop size is determined by a competition between fluid inertia and surface tension, which allows for the prediction of the drop size from the Weber number and geometry of the nozzle. Once rescaled with the average drop size, the size distribution is found to be described by a compound gamma distribution with two parameters, $n$ and $m$, with the former setting the ligament corrugation and the latter the width of the ligament size distribution. Fit values for $m$ indicate that nozzles of a conical type produce ligaments of almost equal size, while the flat fan nozzles produce broader distributed ligament sizes. Values for $n$ show that, for all nozzles, ligaments are very corrugated, which is not unexpected for such spray formation processes. By using high-speed photography of the sprays, the parameters $m$ and $n$ can be directly measured and, indeed, govern the drop-size distribution.

DOI: 10.1103/PhysRevX.8.031019

\section{INTRODUCTION}

Spraying is one of the most common processes in everyday life; it is important for agriculture, drug administration, printing, firefighting, spray painting, etc. [1,2]. The drop size and drop-size distribution in sprays are of paramount importance for effective spray application; it is important in agriculture pesticide spraying, for instance, that the drop sizes are small for a good deposition and coverage [3], but not too small because of the environmental hazards of airborne spray drift [4-6]. For drug delivery [7-9], one needs small drops that can easily be inhaled, but not so small that evaporation is almost instantaneous. Much work has been done on the optimization of drop sizes in sprays, but most research so far only deals with a few specific aspects of the droplet formation, and a more complete and generic understanding is lacking.

The formation of droplets in sprays ultimately results from the breakup of liquid ligaments, which are often themselves transitorily formed during the destabilization of jets or sheets [10]. In one of the first works that appeared on this topic, Dombrowski and Fraser [11] gave an extensive

Published by the American Physical Society under the terms of the Creative Commons Attribution 4.0 International license. Further distribution of this work must maintain attribution to the author(s) and the published article's title, journal citation, and DOI.
Subject Areas: Fluid Dynamics

qualitative description of liquid sheets produced by flat fan nozzles by using various types of fluids and found several important factors, among which are viscosity, surface tension, pressure, and turbulence in the nozzle. Later works [12-15] provided a more fundamental understanding of sheet breakup and of the microscopic mechanisms at play [10]. In particular, it was understood that one of the main breakup mechanisms for nozzles is due to waves on the surface of the sheet that are produced by friction with the surrounding air. These waves, which were first described by Squire [14], grow in amplitude, causing thickness modulations of the sheet. The modulations will cause the sheet to thin to such an extent that it will rupture, creating sheet fragments of a well-defined size, the Squire wavelength. These fragments will contract to form ligaments, which subsequently break up into droplets. The Rayleigh-Taylor instability [16] describes the instability of the sheet accelerated perpendicular to its plane, which then forms ligaments. These ligaments then break into droplets through a Rayleigh-Plateau instability driven by surface tension [17]. Depending on the initial corrugation of the ligaments, this instability is more or less noisy, producing droplets more or less distributed in size [10]. A similar sequence of events governs the breakup of a liquid jet, as described in Ref. [18] for the specific case of a circular jet with a coaxial air flow. Although, in some cases, the mechanisms of spray formation are reasonably understood, a systematic comparison of the drop size and size 

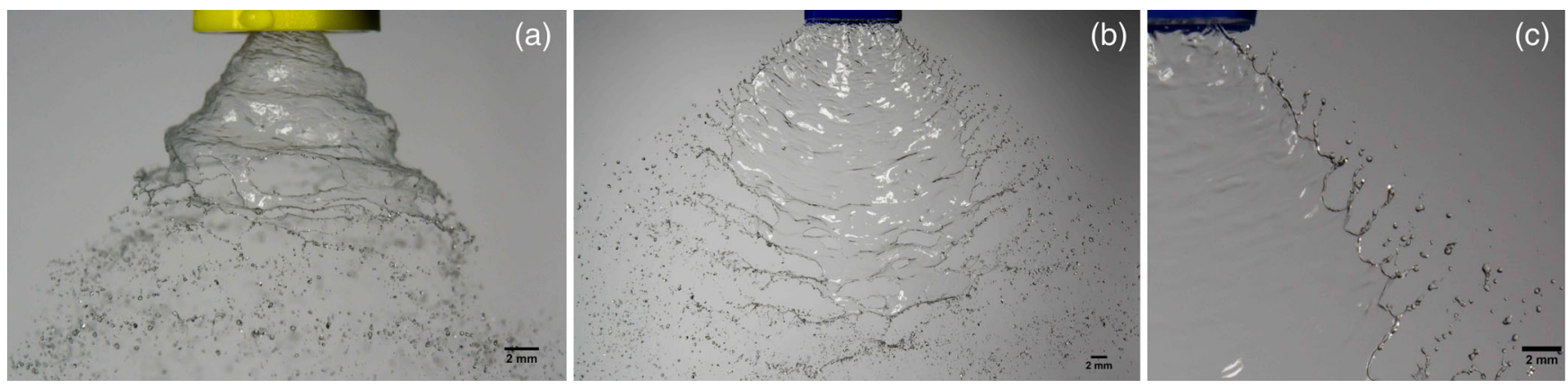

FIG. 1. (a) Picture of the conical nozzle with an operating pressure of 2.0 bar. The Squire wave is clearly visible, with a wavelength in agreement with the prediction. (b) The flat fan nozzle at 2.0 bar, with flapping and breakup occurring near the bottom of the picture. (c) Zoomed-in picture of the flat fan nozzle at low pressure (1.0 bar), showing droplets coming from the sheet's rim that are of the order of the nozzle size.

distribution from liquid sheets produced in different geometries and with different liquids is still lacking.

In this paper, we examine the spray formation as a function of all relevant parameters: nozzle type, spraying pressure, and fluid properties. We show that the drop size can be predicted from the Weber number (which gives the competition between fluid inertia and surface tension) and the geometrical properties of the spray nozzle. Our findings allow one to directly calculate the drop size as a function of all of these parameters. In addition, the size distribution is found to be mostly universal, implying that all distributions for different parameters collapse when scaled with the mean drop size. Therefore, the results presented here allow us to obtain the drop size and its entire drop-size distribution without adjustable parameters.

We study the breakup of flat or conical liquid sheets formed with standard spraying nozzles used in many applications; together these cover a large fraction of all spraying applications. The difference is that the flat fan nozzle forms a flat liquid sheet that breaks up, as in Figs. 1(b) and 1(c), whereas for the conical nozzle, the sheet that emerges from the nozzle is cone shaped [Fig. 1(a)]. Such spray nozzles have a round or oval inlet opening, after which the fluid is pushed out through the outlet that is either wedge shaped or circular; here, the sheet is formed and subsequently breaks up to form the spray. To determine the droplet size distribution, a laser diffraction method (Malvern Spraytec) is used. An expanded laser beam is passed through the spray, and the diffraction pattern is measured with a 2D chargecoupled device array. The diffraction angle is inversely proportional to the size of the droplet, so the light diffraction pattern allows us, assuming a spherical shape of the droplets, to obtain the droplet size distribution. The laser beam is placed $40 \mathrm{~cm}$ below the nozzle, where, for all nozzles, pressures, and fluid parameters, no further breakup occurs. We verified using high-speed photography that, at this distance from the nozzle, drops are indeed spherical, with few exceptions for large droplets. To investigate the possible effect of droplets having a nonspherical shape due to an oscillating motion, we looked at the droplet size distribution at different heights, since one expects shape oscillations to damp out, and saw no difference. The pump pressure was varied between 1.0 and 5.0 bar, and five different standard spray nozzles were used; see Appendix A for details about the nozzles.

\section{EXPERIMENTS}

First, the flow rate was measured for the different nozzles as a function of the pressure to determine an effective hydraulic area $A_{\text {hyd }}$, from which a characteristic nozzle inlet dimension can be extracted (see Appendix B). We then measured the droplet size and the droplet size distribution as functions of all relevant parameters. The most common way to characterize the droplet size is by the volume median diameter, $D_{50}$, as, for example, in the applications of pesticides [19] or droplet size predictions [13]. Other less common definitions are Sauter mean diameter, $D_{32}$, or the arithmetic mean, $D_{10}$. Although all these parameters provide a measure for the droplet size, any assessment on the spray should always take the whole size distribution into account. One should, e.g., be careful comparing characteristic droplet sizes if the distributions change shape significantly.

As a first variation of parameters, we changed the operating pressure for the different nozzle types. The droplet size distributions for a flat fan nozzle are shown in Fig. 2(a) for a range of pressures. It can be observed that the higher the pressure, the more the drop-size distribution shifts to smaller drops and, thus, a smaller median drop size. Quantitatively, the median drop size shows a power law dependence on the flow rate $q$ with a power of $2 / 3$ that is valid for all nozzles tested here. Moreover, all data collapse on a single line when the $D_{50}$ is divided by the effective nozzle area (Fig. 3), i.e.,

$$
D_{50} \sim A_{\text {hyd }} q^{-2 / 3} \text {. }
$$

To investigate the effect of the physical properties of the spraying liquid, we varied both the viscosity and the surface tension of the spraying liquid. The viscosity was varied by 

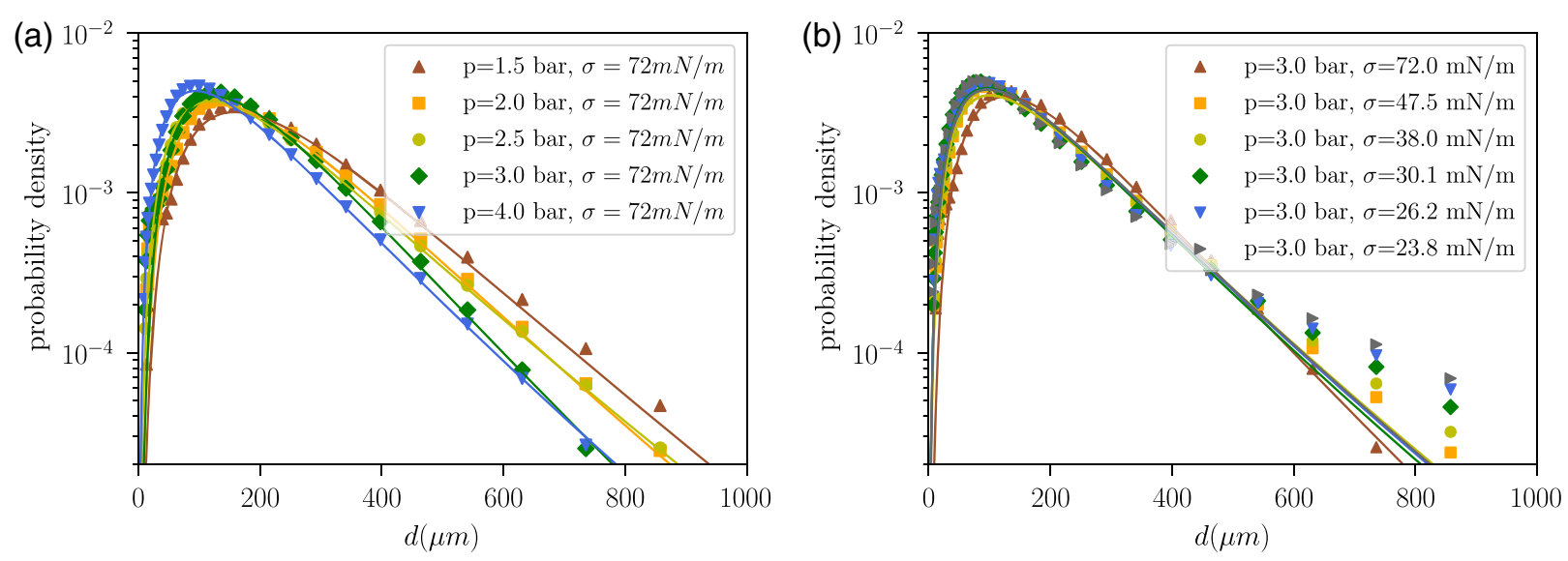

FIG. 2. (a) The droplet size distribution for pure water with pressures from 1.5 to 4.0 bar for the Teejet 110-03 nozzle (flat liquid sheet). Fit lines are of the global size distribution [Eq. (11)] with parameters $m$ and $n$ around 5. (b) Droplet size distributions of water-ethanol mixtures with different surface tensions (Teejet 110-03, flat liquid sheet). The fit parameters are similar to those for pure water. A deviation from the fit lines can be seen for large droplet sizes, with a systematic enhancement for increasing ethanol concentrations.

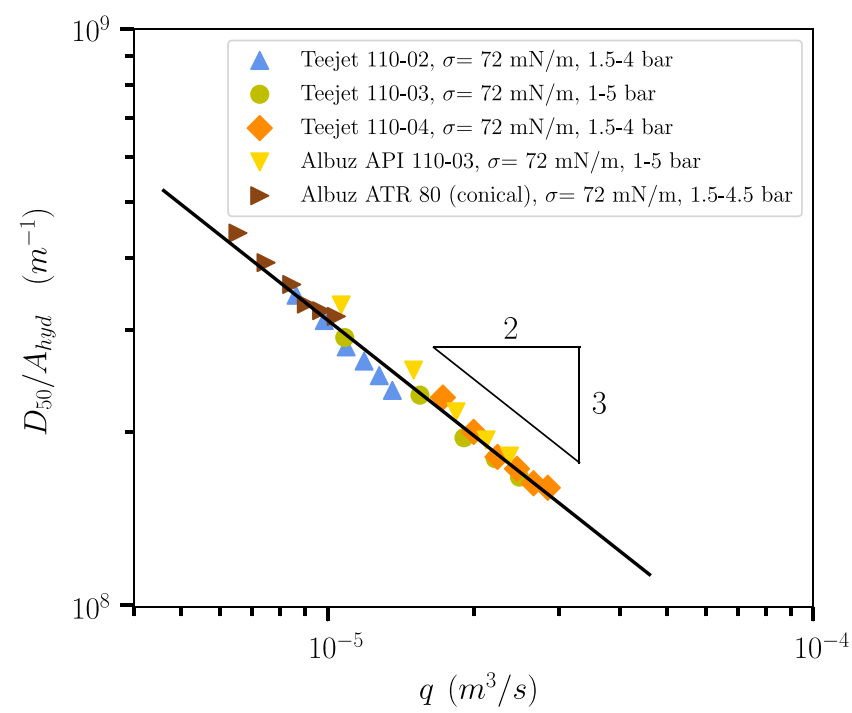

FIG. 3. A log-log plot of $D_{50}$ divided by the effective nozzle area against the flow rate for all nozzles. The fit line has a slope of $-2 / 3$.

as much as a factor of 30 by using water-glycerol solutions, with viscosities ranging from 1 to $32 \mathrm{mPa} \cdot \mathrm{s}$, which covers most spraying liquids used in practice. Even with this significant change in viscosity, the median droplet sizes are found to be unchanged within the experimental accuracy over this range (see Appendix C). The distributions show a slight change for the highest viscosities (Fig. 4); this effect is, however, very small.

The surface tension $\sigma$ was varied between approximately 23 and $72 \mathrm{mNm}^{-1}$ by using water-ethanol mixtures of different concentrations. The water-ethanol mixtures are an appropriate way of probing the effect of the surface tension, since the viscosity is relatively unchanged, as well as the density of the fluid. Moreover, the breakup happens at a timescale of the order of $1 \mathrm{~ms}$, so no surface tension gradient effects are to be expected. This is in contrast with

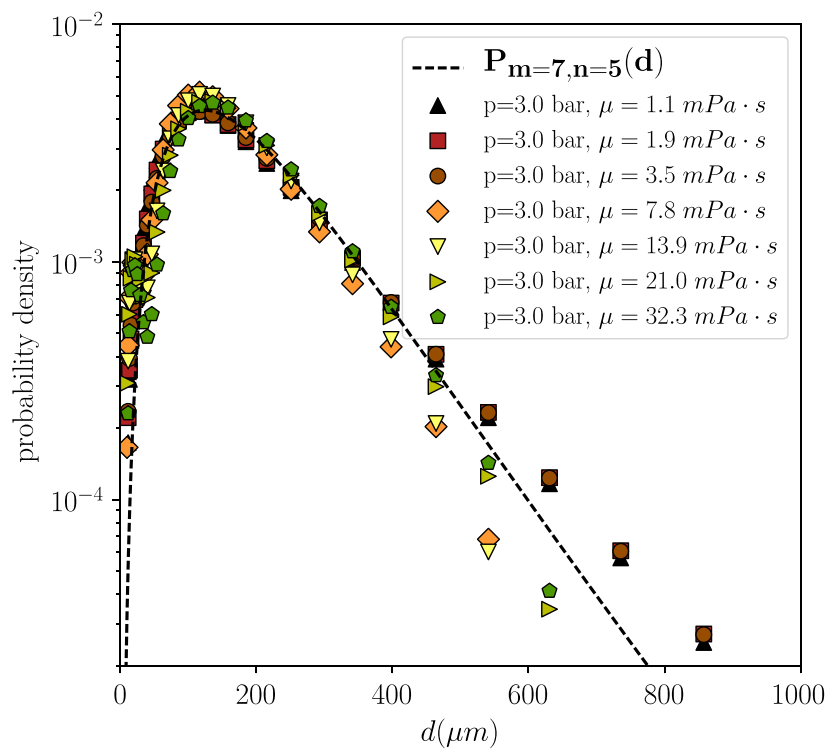

FIG. 4. Droplet size distribution of water-glycerol mixtures, with viscosities ranging from $\mu=32.3$ to $1.1 \mathrm{mPa} \cdot \mathrm{s}$ (Teejet 110-03). For this range of viscosities, there is no significant change in the size distributions.

the use of surfactants to lower surface tension, which are completely ineffective due to the relatively slow dynamics of the surfactant molecules. Measured droplet size distributions of pure water and water with a "fast" surfactant such as SDS [20-22] around the CMC are found to be indistinguishable (see Appendix C).

Visual observation using a high-speed camera shows a difference in sheet breakup dynamics upon a change in surface tension: The spray with lower surface tension appears to be more unstable, with the ligament formation starting closer to the nozzle and more rapid droplet formation. In accordance with this visual observation, the peak of the droplet size distribution is shifted to smaller 
diameters with lower surface tensions. This effect is systematic, but turns out to be rather small over the limited range over which the surface tension can be varied, with a maximum change of $20 \pm 6 \%$.

Interestingly, a consistent deviation from the fit lines can clearly be seen at large droplet sizes with increasing ethanol concentrations [Fig. 2(b)]. The exact origin of this deviation remains unclear, but could be due to an enhancement of coalescence events caused by Marangoni stress gradients.

\section{MEDIAN DROP SIZE}

We are now in a position to develop a general relation between the drop size and the spraying and fluid parameters. It was established that the droplet size is insensitive to the liquid viscosity, which is not unexpected when the drop formation results from a competition between fluid inertia and surface tension. For the working range of pressures, the flow speeds in the nozzle are of the order of $20 \mathrm{~m} / \mathrm{s}$, implying that the nozzles operate in a regime where the Squire instability will occur; i.e., the liquid sheet makes a flapping motion due to interaction with the surrounding air [14]. We therefore expect to have two relevant dimensionless numbers:

$$
\alpha=\rho_{\text {air }} / \rho_{\text {liquid }} \text { and } W e=\rho_{\text {liquid }} v^{2} b / \sigma .
$$

Here, $\alpha$ is a density ratio, $W e$ is a Weber number that reflects the force balance, $\sigma$ is the surface tension, $v=q / A_{\text {hyd }}$ the liquid velocity, and $b$ is the characteristic length, which is the minor axis of the elliptical opening of the flat fan nozzle and can be directly calculated from the hydraulic area, since $b^{2} \sim A_{\text {hyd }}$ (see Appendix B for $b$ for the conical nozzle).

The observed dependence of $D_{50}$ on the flow rate directly suggests that

$$
\frac{D_{50}}{b} \sim W e^{-1 / 3} .
$$

To derive this relation and to find the correct dependence on $\alpha$, let us start by defining the breakup length $\ell$ as the radial distance from the nozzle where the sheet starts to break up in distinct pieces. The sheet thickness decays with $\ell$ as

$$
h \sim \frac{b^{2}}{\ell},
$$

since the liquid velocity is constant along the sheet. In addition to the sheet thinning due to the expansion of the liquid film, there will also be thickness modulations induced by the Squire wave on the sheet, where the sheet will be thicker at the crests than at the points of zero amplitude [see Fig. 5(b) for an illustration for the flat fan nozzle]. The sheet will break once the instability has had enough time to grow, i.e., $\tau \sim \ell / v$, where the characteristic timescale $\tau$ can be written as [15]

$$
\tau \sim \frac{\sqrt{\lambda h}}{v \sqrt{\alpha}},
$$

and the Squire wavelength $\lambda$ is given by [14]

$$
\lambda \simeq \frac{4 \pi \sigma}{\rho_{\text {air }} v^{2}} .
$$

Combining these relations, one expects the breakup length to scale as

$$
\ell \sim \tau v \sim b \alpha^{-2 / 3} W e^{-1 / 3}(4 \pi)^{1 / 3} .
$$

The dependence on We was verified by the use of highquality photographs of the sprays for different pressures [Fig. 5(c)].

Since the median droplet size is proportional to the diameter of the ligaments, and the ligaments are formed by pieces of sheet that have a size of the order of the wavelength $\lambda$ [Fig. 5(a)], mass conservation sets the droplet size as

$$
D_{50} \sim \sqrt{\lambda h},
$$

so that finally we arrive at the formula for the droplet size,

$$
D_{50}=C b \alpha^{-1 / 6} W e^{-1 / 3},
$$

(a)

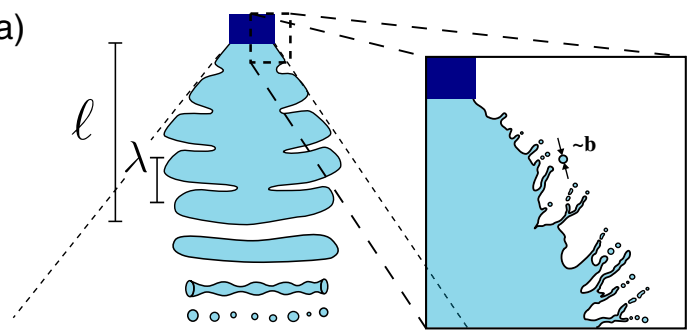

(b)

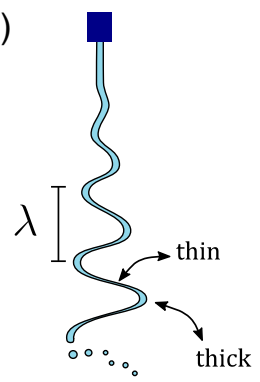

(c)

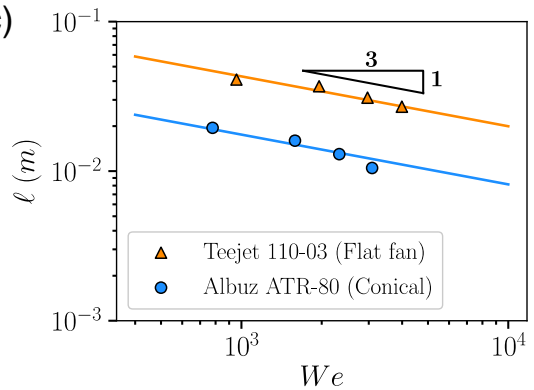

FIG. 5. Schematic representation of the breakup mechanism for the flat fan nozzle. The front view (a) shows how pieces of the sheet of the order of the Squire wavelength rupture from the main sheet to form ligaments at a distance of $\ell$. The ligaments are due to the Rayleigh-Taylor instability and break up into droplets. The side view (b) displays the thickness modulations caused by the increase in amplitude of the Squire wave that eventually lead to the breakup of the sheet. In panel (c), we plot the breakup distance $\ell$ as a function of the Weber number for the Teejet 110-03 (flat fan) and Albuz ATR-80 (cone) nozzle. Distances were obtained by analysis of high-quality pictures of the sprays for different pressures. The fit line shows a $-1 / 3$ power law. 


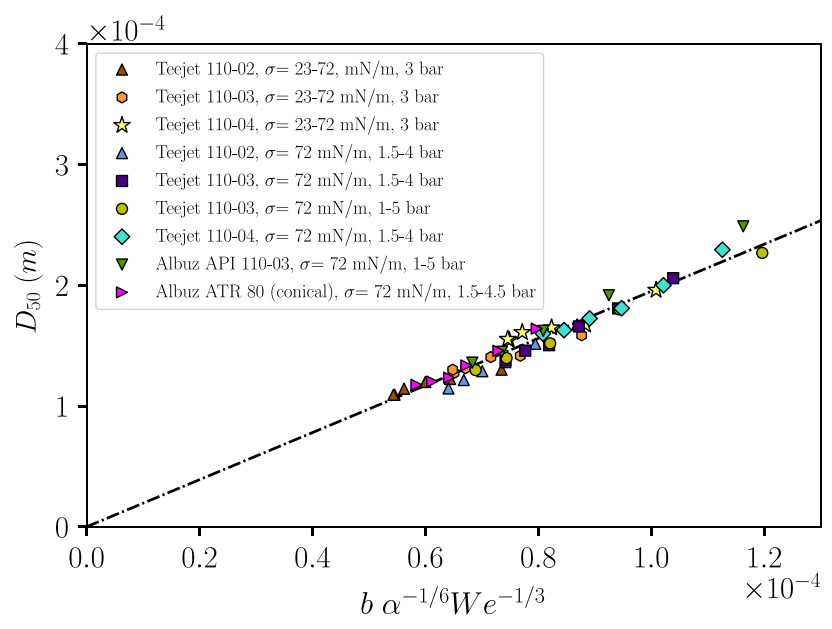

FIG. 6. The median droplet size, $D_{50}$, plotted as indicated in Eq. (9) for all nozzles and various pressures and surface tensions. The slope $C$ is approximately 1.95 .

where $C$ is a dimensionless constant. The data for all nozzles, spraying liquids and pressures are plotted according to Eq. (6) in Fig. 6, which leads to a data collapse on the line with slope $C=1.95$, a constant of order unity. The Weber number at the scale of the droplets is of order $W e_{\text {drop }} \approx 1200$; the above equation then suggests $C \approx 1.9$, a similar value as the slope of Fig. 6. Thus, these arguments allow us to determine the drop size without any adjustable parameters.

Note that the above analysis holds for droplets that are being formed in the center zone of the spray, through the Squire flapping mechanism. The flat fan nozzles, however, suffer from edge effects, with droplets that directly detach from the sheet's rim [Fig. 1(c)]. These droplets, which scale approximately as $b$ [23], are not included in the droplet size measurements and are discussed in more detail in Sec. VI.

\section{DROP-SIZE DISTRIBUTION}

The previous section has explained the scaling of the drop sizes with the operating parameters, but says nothing about the dispersion of the sizes around the mean, a feature that is nevertheless crucial in many applications. Villermaux [10] has emphasized that gamma distributions are significantly better than either the Poisson distribution (random breakup) or the log-normal distribution (a sequence of random processes) for fitting drop-size distribution data, such as those in Ref. [23]. For sprays of ligaments of similar size, they found that the rescaled distribution is best described by the gamma function

$$
\Gamma(n, x=d /\langle d\rangle)=\frac{n^{n}}{\Gamma(n)} x^{n-1} e^{-n x},
$$

where $\langle d\rangle$ is the average droplet diameter and $n$ is a parameter set by the ligament corrugation. Very corrugated ligaments correspond to $n \approx 4-5$, while the most smooth ligaments would lead to $n=\infty$. Figure 7 shows an example of ligament formation due to the appearance of a hole.

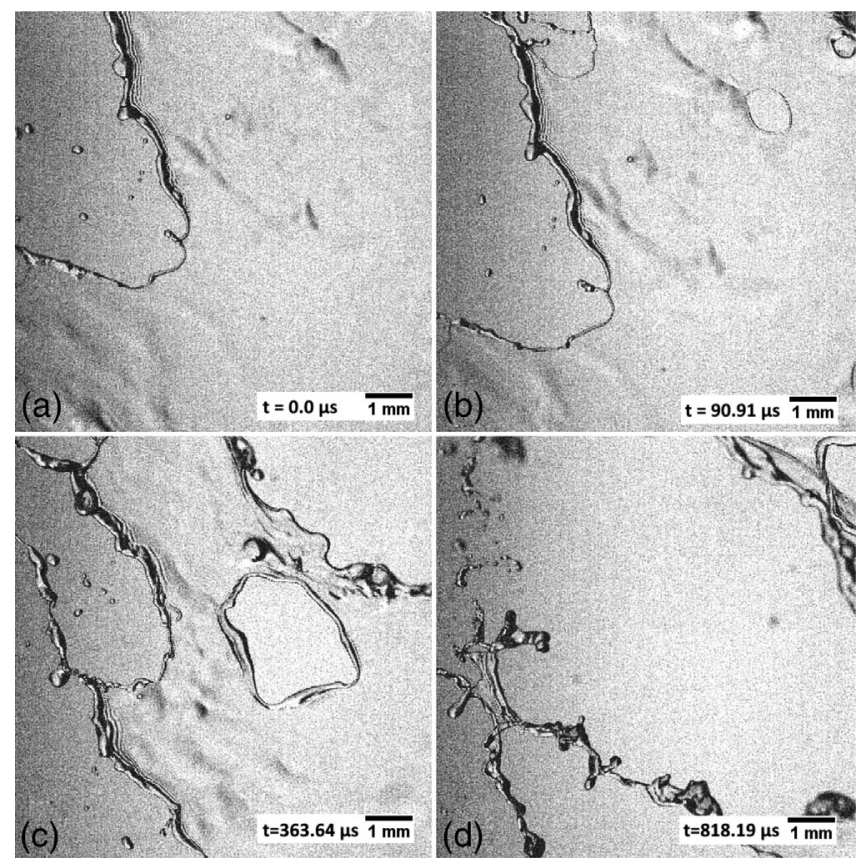

FIG. 7. The formation of ligaments for a flat fan nozzle. At first, there appears a wrinkle on the surface of the sheet (a), followed by the creation of a hole (b), with the subsequent expansion of the hole (c) and formation of ligaments (d).

It should however be stressed, that the main breakup mechanism for the investigated nozzles is not by the formation of circular holes, but by sheet breakup as described above (Fig. 5). For the sprays produced by nozzles, it is expected that ligaments of different diameters are formed whose sizes are also gamma distributed. As shown in Ref. [24], the global droplet size distribution can then be described by the two-parameter compound gamma distribution

$\mathcal{P}_{m, n}\left(x=\frac{d}{\langle d\rangle}\right)=\frac{2(m n)^{\frac{(m+n)}{2}} x^{\frac{(m+n)}{2}-1}}{\Gamma(m) \Gamma(n)} \mathcal{K}_{m-n}(2 \sqrt{m n x})$,

with $\mathcal{K}$ the modified Bessel function of the second kind. The parameter $m$ sets the order of the ligament size distribution and $n$ the ligament corrugation. For the measurements in Fig. 2 for various pressures and surface tensions, we show that the distributions can be rescaled using the mean droplet size $\langle d\rangle$. The data collapse shows that one can change the mean drop size by varying the pressure or surface tension without significantly changing the shape of the distribution [see Fig. 8(a)]. The plotted global distribution $\mathcal{P}_{m=4, n=5}$ shows that, for the flat fan, both the ligament sizes and the ligament corrugation have a very broad distribution. The rescaled distribution for the conical nozzle [Albuz ATR 80, Fig. 8(b)], however, is much narrower distributed, with $m=$ 100 and $n=5$. This indicates that, in contrast with the flat fan nozzle, the conical nozzle has ligaments of uniform size (with $m$ essentially infinite). Not well visible on the graphs are the smallest droplets that appear to be underestimated by 

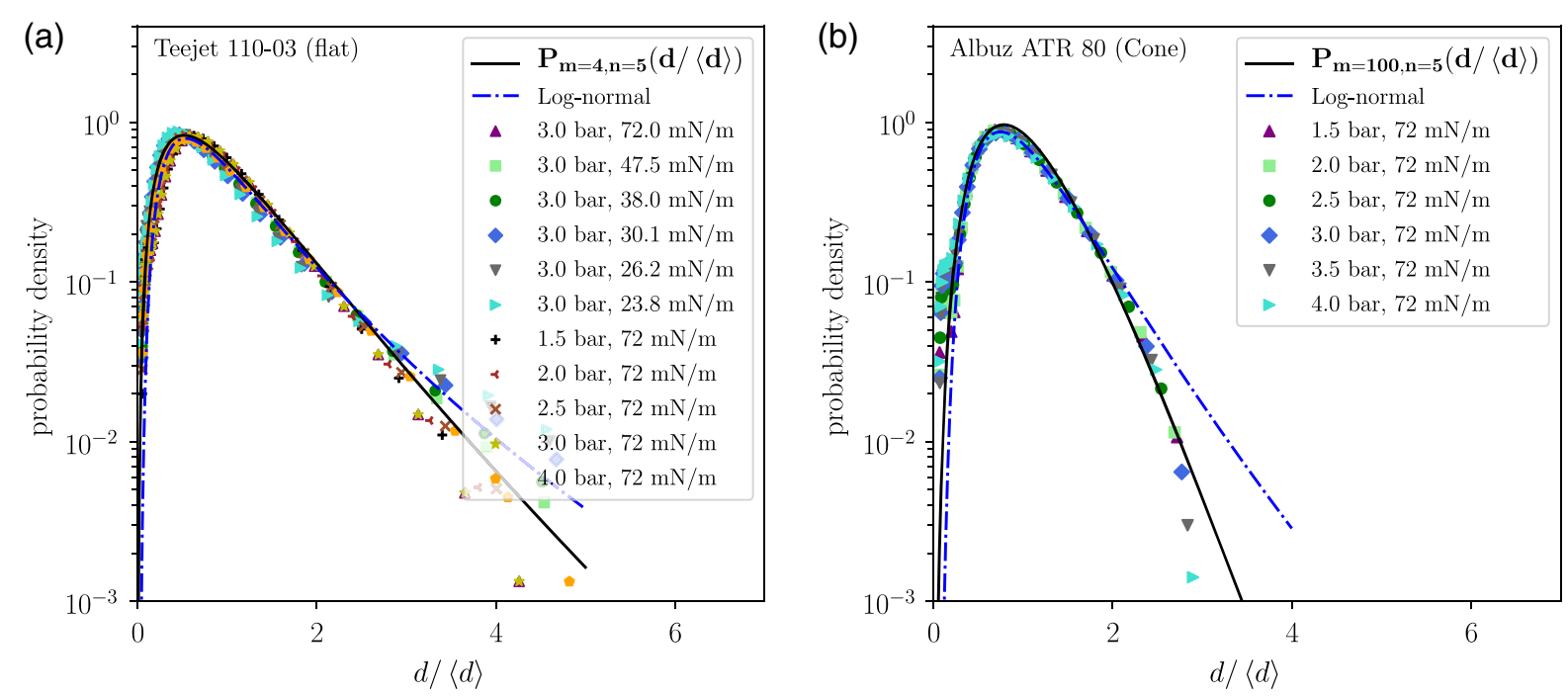

FIG. 8. The rescaled droplet size distributions of pure water for various pressures and water ethanol mixtures for the Teejet 110-03 nozzle (flat liquid sheet) (a) and the Albuz ATR 80 (conical liquid sheet) (b). The data collapse shows that one can change the mean (or median) droplet size by changing the surface tension or pressure, without altering the overall shape of the distribution, but that the shape depends on the type of nozzle [see dashed line in (b) for comparison]. Fit lines show that the distributions can be well fitted by the global size distribution [Eq. (11)] and fit parameters indicate that the conical nozzle produces ligaments of equal size, in contrast with the flat nozzle with a broad distribution of ligament sizes.

the fit, which is likely an experimental artifact due to a recirculation of these droplets in the spraying chamber.

It is worthwhile to note that the log-normal distribution provides a reasonable fit to the distributions as well (Fig. 8), especially close to their maximum (but systematically overestimates their tail), which is the reason why this distribution is popular in the spray community, although the use of this distribution lacks a physical justification [10]. For the log-normal distribution, we have

$$
P\left(x, D_{50}, \theta\right)=\frac{1}{x \theta \sqrt{2 \pi}} e^{-\left[\log \left(x / D_{50}\right)\right]^{2} / 2 \theta^{2}},
$$

with $x$ the droplet diameter, $D_{50}$ the median droplet diameter, and $\theta$ a fit parameter related to the width of the distribution; all distributions can be rescaled with the median $D_{50}$. This suggests that, given a certain nozzle, the fit parameter $\theta$ should be the same and is, thus, independent of pressure or surface tension. Although the shape of the distributions seems to be equal for the range of pressures and surface tensions, there is a slight dependence of $\theta$ on the spraying parameters (see Appendix E).

\section{LIGAMENT SIZES AND CORRUGATION}

Fit parameters $m$ and $n$ of the droplet size distribution of the Teejet 110-03 (flat fan nozzle) and Albuz ATR 80 (conical nozzle) indicate a significant difference in the distribution of ligament sizes between the two nozzle types. To directly measure the values of these parameters, we made high-quality pictures (NIKON D5200) of the sprays by using a fast flash light (Vela One) with a $5-\mu$ s flash duration. For easier analysis, we compared the two nozzles at a low operating pressure of 1.0 bar. The values of the parameters are determined as in Ref. [25], taking into account that ligaments that have a larger diameter to destabilize slower according to $\tau \sim \sqrt{\rho d^{3} / \sigma}$, causing a sampling bias that we corrected for. The ligament corrugations are defined as $n=1 /\left(\left\langle d_{c}^{2}\right\rangle /\left\langle d_{c}\right\rangle^{2}-1\right)$, where $d_{c}$ are the diameters of the inscribed circles of a ligament [Fig. 9(b)]. For smooth ligaments, the diameters would be all the same, leading to $n=\infty$. If the ligament is, however, very corrugated, the diameters are very dissimilar, which results in $n \approx 4-5$ in the maximally corrugated case. Figure 9 shows an example measurement of a ligament of the conical nozzle, which results in a ligament corrugation of $n=6.5$ and a mean diameter $\left\langle d_{c}\right\rangle=l=0.13 \mathrm{~mm}$. Similarly, the parameter $m$ is defined as $m=1 /\left(\left\langle l^{2}\right\rangle /\langle l\rangle^{2}-1\right)$, with $l$ the mean diameters of different ligaments. For a reasonable estimate of the parameters, only ligaments that have a sufficient length are included in the analysis. Furthermore, for ligaments that have multiple branches, the connected ligaments that already destabilized are considered not to be part of the (main) ligament. In practice, a lot of ligaments have thinner side branches that have already partially broken up into droplets and are, therefore, not included in the analysis. Ignoring those side branches makes the ligaments appear less corrugated, causing $n$ to be slightly overestimated. For the conical nozzle, we find that, on average, $m=60$ and $n=5.9$, and for the flat fan nozzle, $m=9.5$ and $n=5.7$. Even though visual evaluation of the pictures clearly shows that the ligaments are very corrugated (i.e., $n \simeq 5$ ), this is, in fact, a slight overestimation of the values of $n$. This can be attributed to the previously mentioned selection of ligaments that are used for the analysis, and the fact that the pictures are a $2 \mathrm{D}$ representation of a $3 \mathrm{D}$ 

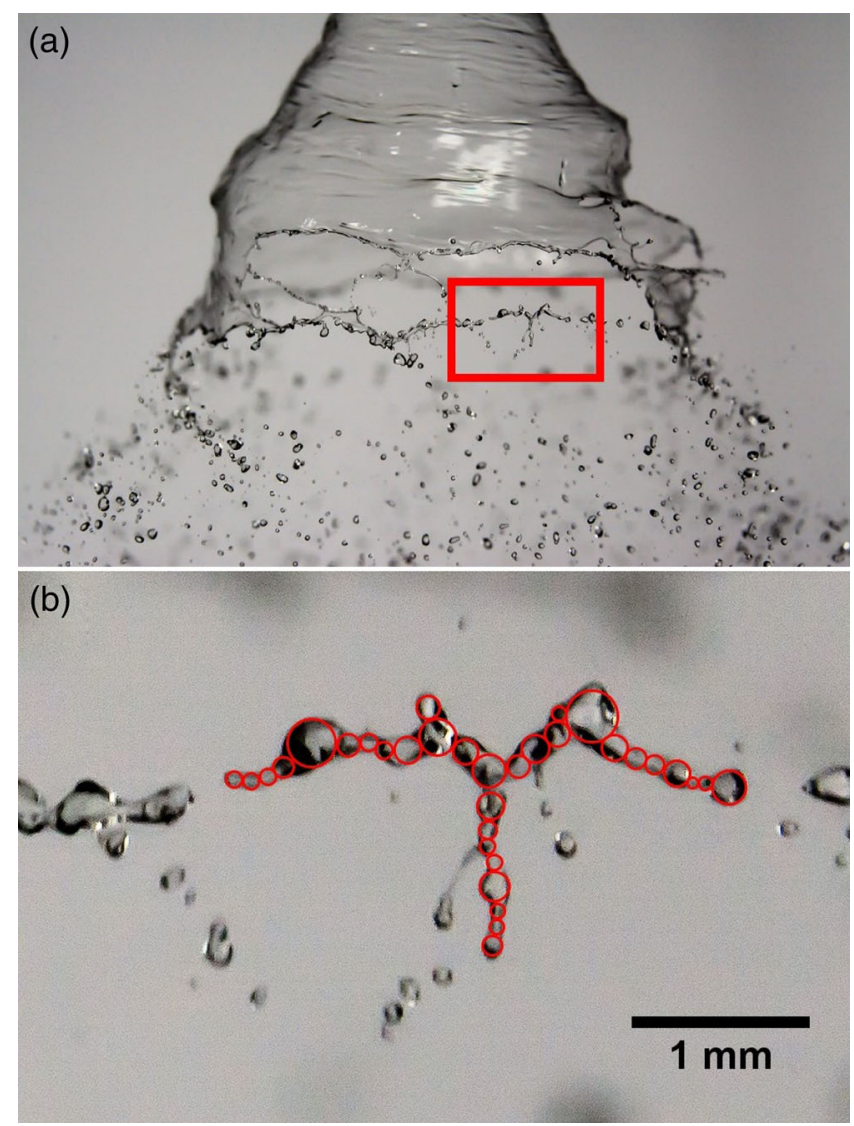

FIG. 9. (a) Conical nozzle (Albuz ATR 80) at a pressure of 1.0 bar, with (b) a zoomed-in selection indicated by the red frame. Inscribed circles allow for an estimation of the ligaments corrugation $n$ and mean diameter $\left\langle d_{c}\right\rangle$ as described in Ref. [25], which, in this case, yields $n=6.5$ and $\left\langle d_{c}\right\rangle=0.13 \mathrm{~mm}$.

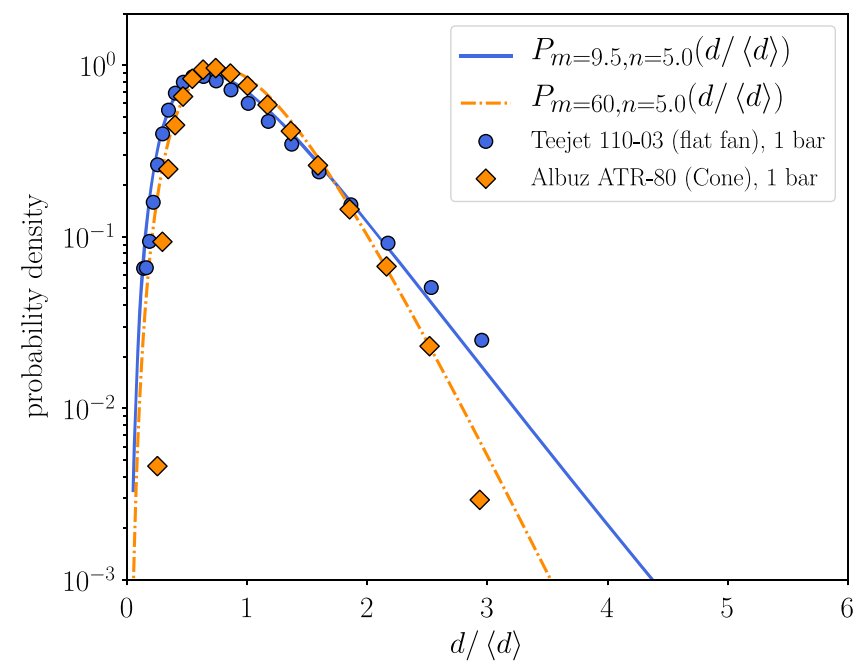

FIG. 10. Comparison of the droplet size distribution of the Teejet 110-03 (flat fan) and Albuz ATR 80 (conical) nozzle at 1.0 bar. The lines indicate the predicted drop-size distributions according to the measured values of $m$ and setting the values $n=5$. ligament. The drop-size distributions of the two nozzle types at this pressure are plotted in Fig. 10, with the lines indicating the predicted size distribution using the measured values of $m$ and setting $n=5$. The agreement between the prediction and measured distributions clearly confirms how the size distribution is governed by the ligament sizes and corrugation. The high value of $m$ for the conical nozzle indicates that ligaments are of uniform size, while the low value of $m$ for the flat fan nozzle indicates a very broad dispersion of ligament sizes. It should be noted that, at this low pressure (1.0 bar), the values of $m$ are not the same as the values used in Fig. 8, which only includes pressures $\geq 1.5$ bar, leading to $m=100$ and $m=5$ for the conical and flat fan nozzle, respectively. The parameter $m$, thus, reaches its converged value at a pressure of $1.5 \mathrm{bar}$. The shape of the distribution is, therefore, only pressure independent for pressures $\geq 1.5$ bar.

\section{DROPLETS FROM THE RIM}

Droplet sizes and droplet-size distributions for the flat fan nozzles are measured along the center of the spray. Still, a significant amount of droplets are formed on the sheet's rim as shown in Fig. 1(c) and illustrated in Fig. 5(a). By collecting the droplets that came from the edge of the sheet, the contribution of this part of the spray could be determined. Then, by comparing this value with the total flow rate, it is estimated that these droplets make up roughly $25 \%$ of the total sprayed volume. Since the droplet sizes from the edge scale with the nozzle size $b$, they are larger on average than droplets from the middle; $D_{50}=371 \mu \mathrm{m}$ compared to $D_{50}=231 \mu \mathrm{m}$ at a pressure of 1.0 bar. This shows that, considering all parts of the spray, the complete droplet-size distribution would, in fact, be broader than the previously shown distributions. It is, however, not easy to combine the two different contributions, since there is no clear distinction between the two different breakup zones, and there is a constant interference with small droplets that are measured outside the targeted measuring zone due to drift.

The size distributions of the droplets from the edge are interesting (Fig. 11). Since ligaments that come from the edge are all of similar size, the distribution is quite narrow and equivalent to the distribution of the conical nozzle, which also produces similarly sized ligaments. Moreover, for a pressure of 1.0 bar, the ligaments are also relatively smooth $(n=10)$, making the distribution even more narrow. However, at higher pressures starting from 1.5 bar, ligaments are already maximally corrugated $(n=5)$, making the shape of the distribution pressure independent from this pressure on, which is similar to what is seen for the main parts of the sprays (see previous section). These observations match the observations done by high-speed photography, wherein it can be seen that, at 1.0 bar, the ligaments are still smooth and equal in size, but at a higher pressure of 2.0 bar, they are very corrugated. It should be noted, however, that the lowest possible value of $n$ is, in fact, $n=4$ [26]; this suggests that ligaments from the rim's edge cannot get as corrugated as in other spray formations such as jets. 


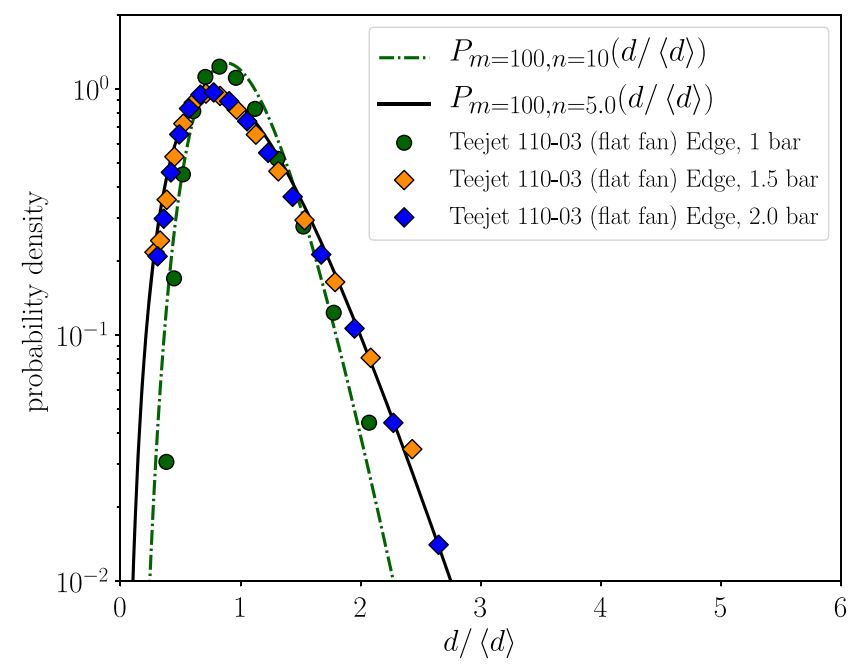

FIG. 11. Droplet size distributions of droplets from the edge of the Teejet 110-03 nozzle (flat fan) with pressures 1.0, 1.5, and 2.0 bar. The ligaments are of uniform size, resulting in $m=100$. For 1.0 bar, the ligaments are still quite smooth with $n=10$, making the distribution more narrow. For pressures $\geq 1.5$ bar, the ligaments are maximally corrugated, resulting in $n=5$.

These results show that there is a sudden change in ligament characteristics with a small increase in pressure. This is not very surprising, considering that these nozzles are used and designed to operate at pressures higher than 2 bar. Actually, if the pressure is too low (below 1 bar), no sheet will be formed. It can, therefore, be expected that the flat fan nozzles only reach a stable operation at $1.5-2$ bar.

\section{DISCUSSION AND CONCLUSION}

We have found that the droplet-size distribution depends on the liquid surface tension, nozzle type, and flow rate of the spray, but it is independent of the viscosity of the liquids used. The average drop size can be changed without altering the overall shape of the distribution significantly by simply changing the nozzle or pressure or by adding ethanol to lower the surface tension. In the latter case, however, there remains an unexplained increase for the biggest droplets, although this increase is not very significant in an absolute sense.

Because of the relatively weak dependence of the median droplet size on the spraying parameters, the most effective and practical way of changing the median droplet size is by changing the pressure and, hence, the flow rate. Changing the surface tension has a limited effect, since for most fluids, the surface tension cannot be changed significantly, without adding a substantial amount of another liquid. Even if the surface tension could be changed by a factor of 3 , keeping other parameters the same, the resulting change in droplet size would be no more than $44 \%$. However, adding another liquid to lower the surface tension often changes the density of the solution as well, which generally has the opposite effect. For the case of ethanol-water mixtures, e.g., adding as much as $80 \mathrm{wt} \%$ ethanol results in a theoretical change of only $36 \%$ in the droplet size due to the variation of $\sigma / \rho$. The measured change is a bit lower, because of an increase of the largest droplets for high ethanol concentrations, which is probably due to enhanced coalescence of droplets driven by Marangoni stress gradients.

The derived scaling law for the droplet size is in accordance with a similar formula obtained by Dombrowski and Johns [13]. Their results contain a correction term for the viscosity that indeed can be neglected for the range of parameters we investigated. Our formula differs in the use of the characteristic nozzle size $b$, calculated from the effective hydraulic area. This approach works surprisingly well and allows us to determine the droplet size even though the nozzle type is very different. Dombrowski and Johns, however, use a nozzletype-dependent (and possibly pressure-dependent) parameter, and they only verify their findings for flat fan nozzles.

Drops in a spray come from the breakup of columnar liquid structures called ligaments [10]. Ligaments may be smooth or corrugated, and a complex spray may be formed from ligaments that are all similar or very dissimilar. The dispersion of the drop sizes in a spray, thus, results from both the dispersion of sizes coming from the breakup of a single ligament (measured here by the parameter $n$ ) and the distribution of the ligament sizes (measured here by $m$ ). In practice, ligament corrugations are large, and the ligaments are not too different, so that the final width of the drop-size distribution is controlled by ligament breakup (see, e.g., Ref. [27]). The present study confirms the existence of this limit (with the conical sheet), but it shows also that dispersion in ligament sizes can contribute to the final structure of the spray (with the flat sheet).

It has been shown that the maximum corrugation possible is $n=4$ [26]. The rescaled distribution of the center of the sprays, as well as droplets coming specifically from the flat fan nozzle's edge, are, however, better fitted with $n=5$, although the differences between $n=4$ and $n=5$ are small. This could indicate that, unlike jets, ligaments produced by spray nozzles do not reach their maximally corrugated state, even at high operating pressures.

Perhaps one of the most interesting findings is a measurable difference in droplet size distributions between the conical and flat fan nozzle. The parameters $m$ and $n$ show that, for both nozzle types, the ligaments are very corrugated, but that, in contrast with the flat fan nozzles, the conical nozzle produces ligaments of surprisingly uniform size. With high-speed photography, this difference could be directly observed. The similarly sized ligaments for the conical nozzle are explained by the fact that, because of the Squire wave and uniform sheet thickness at the breakup zone, almost equally sized pieces of sheet rupture from the main cone, giving similarly sized ligaments. This is not the case for the flat fan nozzle that suffers from boundary effects and has a nonuniform sheet thickness as a result 
of the elliptical opening. For these reasons, the sheet breaks up in irregular ways, creating ligaments that vary strongly in size. Additionally, the flat fan nozzles produce large droplets at the rim of the sheet; these droplets account for roughly $25 \%$ of the total sprayed volume and would make the complete distribution even broader. So whenever narrowly distributed droplets are required, a nozzle of the conical type is preferred.

\section{ACKNOWLEDGMENTS}

This work is part of the Industrial Partnership Program Hybrid Soft Materials that is carried out under an agreement between Unilever Research and Development B.V. and the Netherlands Organization for Scientific Research (NWO). E. V. thanks the "Agence Nationale de la Recherche" for funding of the ANR "FISICS" Grant No. ANR-15-CE30-0015-03. We thank the anonymous referee for pointing out the (anomalous) increase of larger droplets with increasing ethanol concentrations and for providing a possible explanation.

\section{APPENDIX A: NOZZLES}

The opening sizes of the nozzles are measured with a microscope with a magnification of 3.2. First, the height and width of the elliptical opening is obtained, from which the area is calculated (or circular opening in the case of the conical nozzle). The measured area of all the nozzles can be seen in Table I, together with the discharge coefficients. The investigated nozzles are commonly used in agriculture, of which two examples can be seen in Figs. 12 and 13.

TABLE I. The opening area of the different nozzles plus the discharge coefficient. The conical nozzle has a low discharge coefficient due to the complex flow in the outlet.

\begin{tabular}{lcc}
\hline \hline & Area $\left(\mathrm{m}^{2}\right)$ & Discharge coefficient \\
\hline Teejet 110-02 & $5.2 \times 10^{-7}$ & 0.94 \\
Teejet 110-03 & $8.3 \times 10^{-7}$ & 0.94 \\
Teejet 110-04 & $1.1 \times 10^{-6}$ & 0.91 \\
Albuz API 110-03 & $8.8 \times 10^{-7}$ & 0.85 \\
Albuz ATR 80 (cone) & $1.1 \times 10^{-6}$ & 0.34 \\
\hline
\end{tabular}
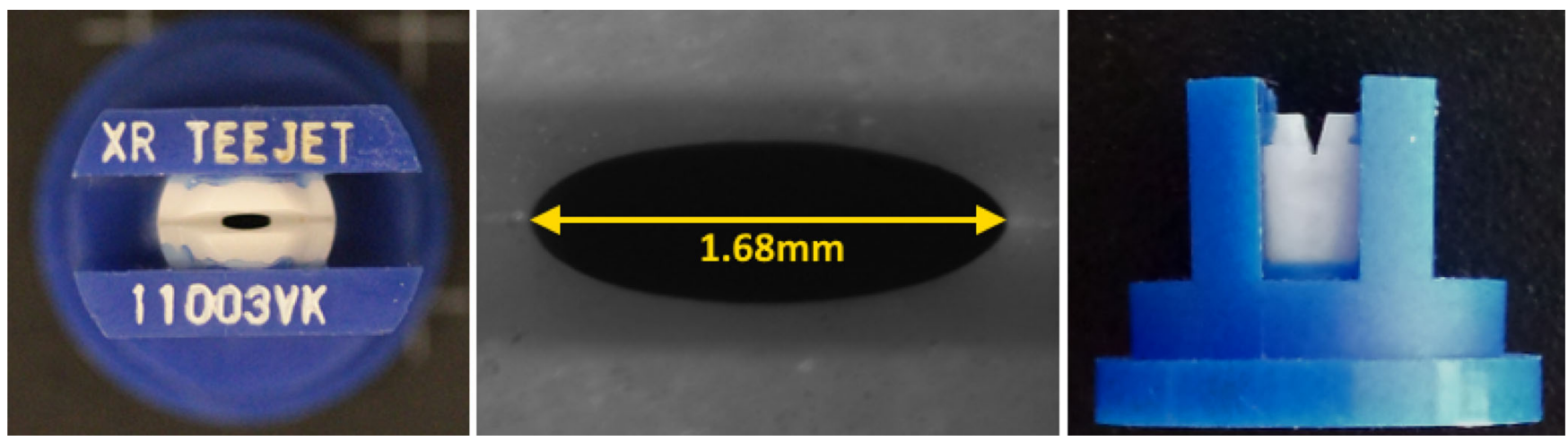

FIG. 12. A picture of the Teejet 110-03 nozzle seen from above, with a magnification of the elliptical opening, plus a side view. This nozzle produces a flat liquid sheet.
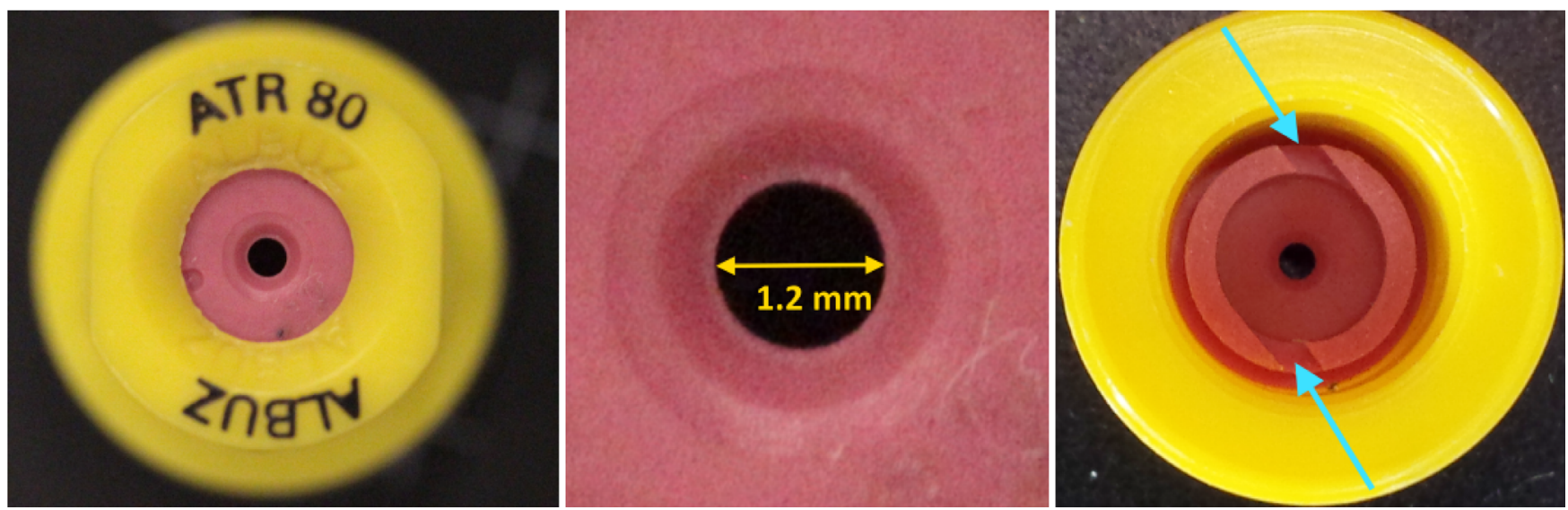

FIG. 13. A picture of the Albuz ATR 80 nozzle that produces a conical liquid sheet, seen from above (left-hand side) and from the inside (right-hand side). The inside of the nozzle has a cover, so that the fluid has to go through the two openings at the edge (blue arrows), forcing the liquid into a vortex flow, after which it leaves the nozzle through the 1.2-mm hole. 
Teejet and Albuz are the brand; the 110 stands for the angle of the spray sheet; and the 02,03 , and 04 stand for the size of the inlet opening. In Fig. 12, there is a top and side view of a Teejet nozzle that produces a flat liquid sheet, and in Fig. 13, one can see a nozzle that produces a conical liquid sheet. The conical nozzle, unlike the flat fan nozzles, has a circular opening, but before the liquid leaves the nozzle, the fluid is forced into a vortex flow, as can be seen on the right-hand side of Fig. 13. This then guides the fluid around the edge of the circular opening, thereby creating a liquid cone instead of a jet.

\section{APPENDIX B: FLOW RATE AND NOZZLE SIZE}

The flow rate $q$ was measured for different pressures using pure water. The Engineering Bernoulli equation gives

$$
\frac{1}{2} \rho v_{\text {after }}^{2}+p_{\text {after }}=\frac{1}{2} \rho v_{\text {before }}^{2}+p_{\text {before }}-l_{v},
$$

where $\rho$ is the density, $v$ the fluid velocity, and $p$ the pressure before and after the nozzle opening; $l_{v}$ denotes viscous losses that typically scale with $\rho v_{\text {after }}^{2}$ [28]. If we write $v=q / A$, where $A$ is the area of the nozzle opening, and assume that the small fluid velocity before the nozzle opening can be neglected with respect to the velocity in the nozzle, one finds $q=C_{d} \cdot A \cdot \sqrt{(2 p / \rho)}$, where $C_{d}<1$, the discharge coefficient, accounts for losses (see Table I).

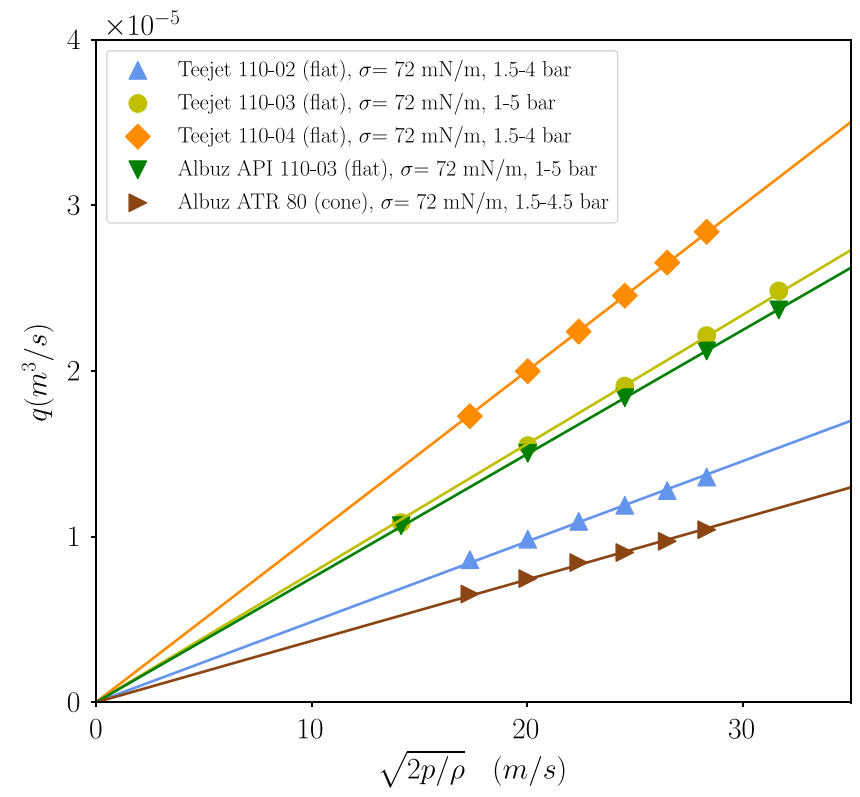

FIG. 14. The flow rate $q$ vs $\sqrt{2 p / \rho}$, where $p$ is the pressure and $\rho$ the density of water. The slope equals the effective area of the opening of the nozzle.
An effective hydraulic area can then be defined as $A_{\text {hyd }}=C_{d} \cdot A$. Figure 14 shows the flow rate $q$ vs $\sqrt{(2 p / \rho)}$; indeed, all data points are on a straight line that goes through the origin. The slope of the line gives the effective hydraulic area of the nozzle. If we then measure the actual area of the inlet opening, we find that the two are very similar, which indicates that the nozzles are designed to minimize entry losses, but also allows us to calculate the hydraulic area from the geometrical properties of the nozzle. The only exception is the conical nozzle that has two small openings within the nozzle that drive a vortex flow, which leads to a discharge coefficient of 0.34 and a small effective hydraulic area. We will, therefore, use the effective hydraulic area for all nozzles.

The characteristic lengths $b$ for the different nozzles were obtained from the effective hydraulic area $A_{\text {hyd }}$. For the flat fan nozzles, with discharge coefficients close to unity, we took $b$ to be the minor axis of the elliptical opening, which, in terms of the effective area, is $b=\sqrt{2 A_{\text {hyd }} / 3 \pi}$, since the major and minor axes have an aspect ratio of $2 / 3$. However, for the conical nozzle, there is no clear-cut measure for the characteristic length $b$ because of its more complicated flow; therefore, as a first approximation, we used the effective area as in the above formula for $b$, which turns out to work well in calculating droplet sizes.

\section{APPENDIX C: SPRAY SOLUTIONS}

Different solutions are used to investigate the influence of the fluid parameters on the droplet size distribution. To determine the flow rate dependence on pressure, water of the spray is collected during a certain time interval, after which the amount of water is measured.

\section{Surface tension}

To vary the surface tension of the spraying liquid without significantly altering the viscosity, water-ethanol mixtures are used. Table II shows the surface tension as a function of the investigated weight percentages as obtained from [29].

TABLE II. Surface tensions of used water-ethanol mixtures.

\begin{tabular}{lc}
\hline \hline wt\% ethanol & Surface tension $\left(\mathrm{mNm}^{-1}\right)$ \\
\hline 0 & 72.0 \\
10 & 47.5 \\
20 & 38.0 \\
40 & 30.1 \\
60 & 26.2 \\
80 & 23.8 \\
\hline \hline
\end{tabular}




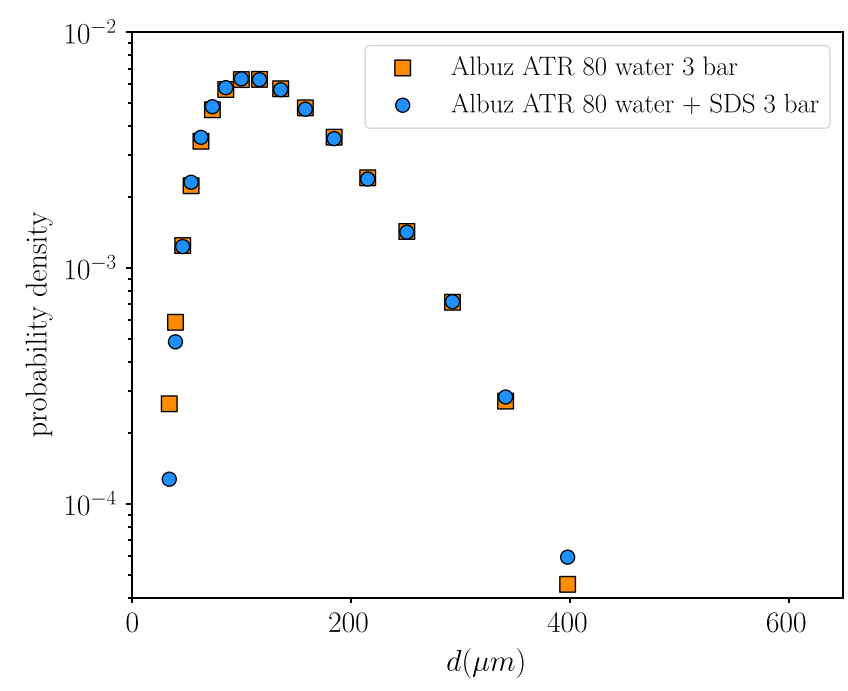

FIG. 15. The size distribution of the Albuz ATR-80 (conical) nozzle at 3 bar with pure water and with a surfactant (SDS) at CMC. There is no visible difference between the two distributions.

Surfactants may seem to be another way of changing the surface tension; however, because of the relatively slow dynamics of the surfactant molecules, they are actually ineffective. Figure 15 shows a measurement of the droplet size distributions of the Albuz ATR-80 (conical) nozzle at 3 bar with pure water and water with SDS at CMC. There is no observed difference between the two distributions, showing that surfactants are unsuitable for changing the surface tension.

\section{Viscosity}

For altering the viscosity of the spraying liquid, waterglycerol solutions are used with viscosities as indicated in Table III. In Fig. 16, the measured median droplet size is plotted against the viscosity for a few nozzles. It is clear from the figure and the distribution in the main text that there is no visible relation between the viscosity and the droplet size.

Table III shows the viscosity obtained through rheology measurements. Glycerol is known to be hygroscopic, and so there is probably a significant amount of water in

TABLE III. Measured viscosities of water-glycerol mixtures.

\begin{tabular}{lc}
\hline \hline wt\% glycerol & Measured value $(\mathrm{mPa} \cdot \mathrm{s})$ \\
\hline 0 & 1.070 \\
20 & 1.860 \\
40 & 3.510 \\
60 & 7.820 \\
72 & 13.900 \\
80 & 21.000 \\
88 & 32.300 \\
\hline \hline
\end{tabular}

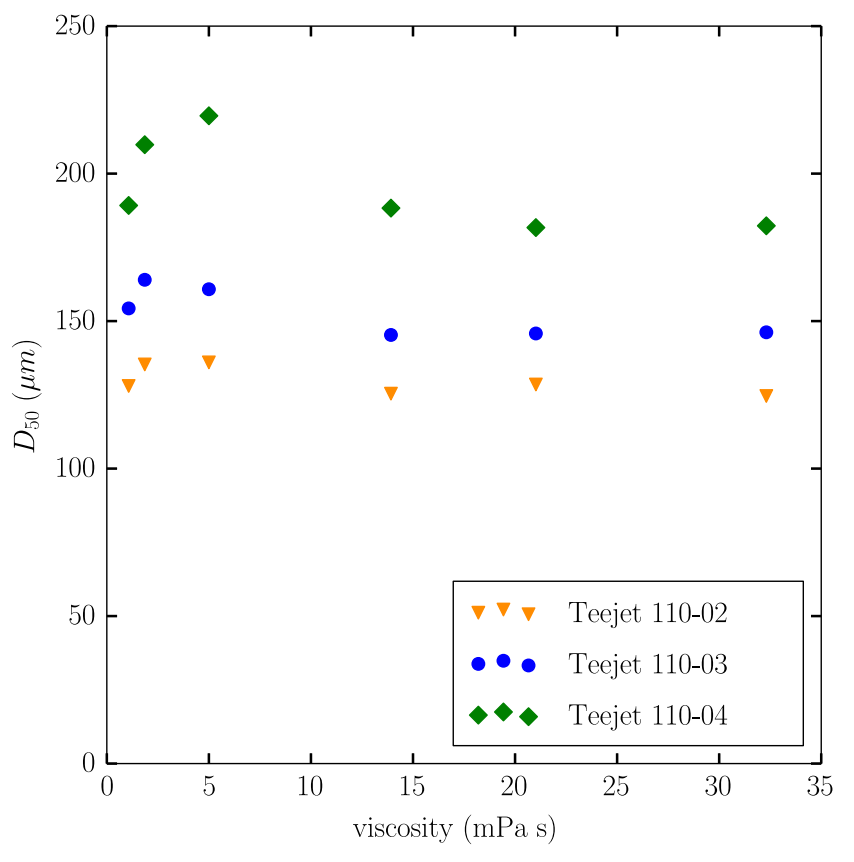

FIG. 16. Median droplet size versus viscosity for three different nozzles. There seems to be no dependency on viscosity for this range.

the glycerol used in the experiments; hence, there is a discrepancy with the literature values.

\section{APPENDIX D: DIMENSIONAL ANALYSIS}

A formula for the median droplet size similar to the one from the main text can be obtained by using dimensional analysis. The droplet size is expected to be insensitive to the liquid viscosity in an inertially dominated regime. The density of the surrounding air is negligible relative to the liquid density and, hence, is not expected to be a primary variable. In that case, the median drop diameter $D_{50}$ should depend only on the liquid density $\rho$; the surface tension $\sigma$; the mean liquid velocity $v$; and a characteristic nozzle length, which we denote $b$. We will return subsequently to the selection of $b$. There are five dimensional variables and three dimensions, so according to the Buckingham pi theorem, there are no more than two independent dimensionless groups. We can take one group as $D_{50} / b$ and the other as $\rho v^{2} b / \sigma$; the second group is a Weber number, which reflects the ratio of inertial to interfacial stresses. We then write

$$
\frac{D_{50}}{b}=f\left(\frac{\rho v^{2} b}{\sigma}\right) .
$$

It is convenient at this point to write $v=q / A$, where $q$ is the volumetric flow rate and $A$ is the true nozzle area, in order to make a direct comparison with the data. Then, Eq. (D1) can be rearranged to 


$$
D_{50}=b f\left(\frac{\rho q^{2} b}{A^{2} \sigma}\right)
$$

$D_{50}$ varies as $q^{-2 / 3}$, so the function $f$ must be a power law with exponent $-1 / 3$, and we obtain

$$
D_{50}=C b^{2 / 3} A^{2 / 3}\left(\frac{\sigma}{\rho}\right)^{1 / 3} q^{-2 / 3}
$$

where $C$ is a constant that may be dependent on nozzle geometry.

We now must select the equipment length scale $b$. For a circular nozzle, the obvious choice is $A^{1 / 2}$, resulting in the proportionality of $D_{50}$ to $A q^{-2 / 3}$. One possibility for ellipses is to use the geometric mean of the major and minor semiaxes; this is equivalent to using the square root of the area and also results in the expected proportionality of $D_{50}$ to $A q^{-2 / 3}$. The choice of $A^{1 / 2}$ is unlikely to be appropriate for ellipses with large aspect ratios or other shapes that are far from a circle, however. A logical choice, which is sometimes used for noncircular nozzles [30,31], is the hydraulic diameter $d_{H}$, defined as $4 A / p$, where $p$ is the wetted perimeter. There is no simple closed-form expression for $p$ for an ellipse, but for ellipses with an aspect ratio of 4 or less $d_{H} \sim A^{1 / 2} \sqrt{r /\left(1+r^{2}\right)}$ to within about $5 \%$, where $r$ is the ratio of major to minor semiaxes. If we use $d_{H}$ for the length scale, we then obtain

$$
D_{50}=C\left(\frac{r}{1+r^{2}}\right)^{1 / 3} A\left(\frac{\sigma}{\rho}\right)^{1 / 3} q^{-2 / 3}
$$

The aspect ratio term varies only between 0.62 and 0.68 for the nozzles used here, so the effect is unlikely to be important, and the result is consistent with the general result that $D_{50}$ is proportional to $A q^{-2 / 3}$. For jets emerging from a coaxial cylindrical nozzle, the proper choice of the length scale $b$ would be the gap spacing, and the scaling would be different.

\section{APPENDIX E: FIT PARAMETERS LOG-NORMAL DISTRIBUTION}

The log-normal distribution is found to give a good fit for the size distributions, although a physical justification is lacking. Still, the fit forms a good tool to assess the form of the distribution. Rescaling the log-normal distribution [Eq. (5)] means that

$$
P\left(x, D_{50}, \theta\right) \rightarrow D_{50} \cdot P\left(x D_{50}, D_{50}, \theta\right)=P(x, 1, \theta) .
$$

So, this rescaling would result in a data collapse if, for all distributions, $\theta$ would be the same. It turns out that one can change $D_{50}$ by changing the pressure or surface tension, without changing $\theta$ very significantly, as shown by Fig. 6 . (Note that $\theta$ does depend on the nozzle type.) Still, $\theta$, which

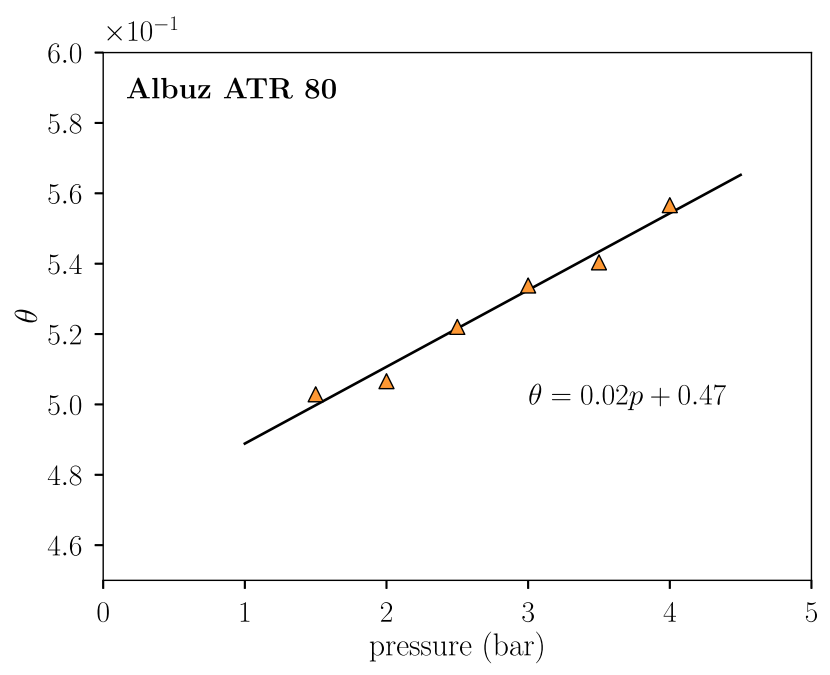

FIG. 17. The fit parameter $\theta$ of the log-normal distribution for the Albuz ATR 80 nozzle as a function of the pressure. The linear relationship shows that the width of the distribution changes with pressure, but the dependency is so weak that the distributions can still be rescaled over this range of pressures. Similar behavior holds for the other nozzles.

sets the width of the distribution, has a very weak dependence on the spraying parameters. As an example, we show the dependency of $\theta$ on the pressure for the Albuz ATR 80 nozzle (Fig. 17). There is a linear relation between $\theta$ and pressure $p$, but the slope is so small that it does not cause any deviations in the data collapse for the range of pressures that we have investigated.

[1] A. H. Lefebvre, Atomization and Sprays (Hemisphere, Boca Raton, 1989).

[2] L. Bayvel and Z. Orzechowski, Liquid Atomization (Taylor \& Francis, London, 1993).

[3] J. R. Lake, The Effect of Drop Size and Velocity on the Performance of Agricultural Sprays, Pesticide science 8, 515 (1977).

[4] S. Reichenberger, M. Bach, A. Skitschak, and H. G. Frede, Mitigation Strategies to Reduce Pesticide Inputs into Ground- and Surface Water and Their Effectiveness: A Review, Sci. Total Environ. 384, 1 (2007).

[5] C. Stainier, M. F. Destain, B. Schiffers, and F. Lebeau, Droplet Size Spectra and Drift Effect of Two Phenmedipham Formulations and Four Adjuvants Mixtures, Crop protection 25, 1238 (2006).

[6] G. Matthews, Pesticide Application Methods (publisher John Wiley \& Sons, New York, 2008).

[7] H. Kublik and M. T. Vidgren, Nasal Delivery Systems and Their Effect on Deposition and Absorption, Adv. Drug Delivery Rev. 29, 157 (1998).

[8] M. B. Dolovich and R. Dhand, Aerosol Drug Delivery: Developments in Device Design and Clinical Use, Lancet 377, 1032 (2011). 
[9] P. P. H. Le Brun, A. H. De Boer, H. W. Frijlink, and H. G. M. Heijerman, A Review of the Technical Aspects of Drug Nebulization, Pharm. World Sci. 22, 75 (2000).

[10] E. Villermaux, Fragmentation, Annu. Rev. Fluid Mech. 39, 419 (2007).

[11] N. Dombrowski and R. P. Fraser, A Photographic Investigation into the Disintegration of Liquid Sheets, Phil. Trans. R. Soc. A 247, 101 (1954).

[12] R. P. Fraser, N. Dombrowski, and J.H. Routley, The Atomization of a Liquid Sheet by an Impinging Air Stream, Chem. Eng. Sci. 18, 339 (1963).

[13] N. Dombrowski and W. R. Johns, The Aerodynamic Instability and Disintegration of Viscous Liquid Sheets, Chem. Eng. Sci. 18, 203 (1963).

[14] H. B. Squire, Investigation of the Instability of a Moving Liquid Film, Br. J. Appl. Phys. 4, 167 (1953).

[15] E. Villermaux and C. Clanet, Life of a Flapping Liquid Sheet, J. Fluid Mech. 462, 341 (2002).

[16] D. H. Sharp, An Overview of Rayleigh-Taylor Instability, Physica (Amsterdam) 12D, 3 (1984).

[17] Lord Rayleigh, On the Instability of Jets, Proc. London Math. Soc. s1-10, 4 (1878).

[18] Ph. Marmottant and E. Villermaux, On Spray Formation, J. Fluid Mech. 498, 73 (2004).

[19] G. Matthews, Pesticide Application Methods (Blackwell Science Ltd., Oxford, 2000).

[20] M. Aytouna, D. Bartolo, G. Wegdam, D. Bonn, and S. Rafaï, Impact Dynamics of Surfactant Laden Drops: Dynamic Surface Tension Effects, Exp. Fluids 48, 49 (2010).
[21] M.-L.E. Timmermans and J. R. Lister, The Effect of Surfactant on the Stability of a Liquid Thread, J. Fluid Mech. 459, 289 (2002).

[22] M. Roché, M. Aytouna, D. Bonn, and H. Kellay, Effect of Surface Tension Variations on the Pinch-Off Behavior of Small Fluid Drops in the Presence of Surfactants, Phys. Rev. Lett. 103, 264501 (2009).

[23] N. Bremond and E. Villermaux, Atomization by Jet Impact, J. Fluid Mech. 549, 273 (2006).

[24] E. Villermaux and B. Bossa, Drop Fragmentation on Impact, J. Fluid Mech. 668, 412 (2011).

[25] E. Villermaux, Ph. Marmottant, and J. Duplat, LigamentMediated Spray Formation, Phys. Rev. Lett. 92, 074501 (2004).

[26] B. Keshavarz, E. C. Houze, J. R. Moore, M. R. Koerner, and G. H. McKinley, Ligament Mediated Fragmentation of Viscoelastic Liquids, Phys. Rev. Lett. 117, 154502 (2016).

[27] H. Lhuissier and E. Villermaux, "Effervescent” Atomization in Two Dimensions, J. Fluid Mech. 714, 361 (2013).

[28] M. M. Denn, Process Fluid Mechanics (Prentice Hall, Englewood Cliffs, NJ, 1980).

[29] D. R. Lide, CRC Handbook of Chemistry and Physics (CRC Press, Boca Raton, Florida, 2005).

[30] P. Sharma and T. Fang, Breakup of Liquid Jets from Noncircular Orifices, Exp. Fluids 55, 1666 (2014).

[31] P. Sharma and T. Fang, Spray and Atomization of a Common Rail Fuel Injector with Non-circular Orifices, Fuel 153, 416 (2015). 